\title{
Téoros
}

Revue de recherche en tourisme

\section{L’attitude des Québécois envers les touristes} Principaux résultats d'un sondage exploratoire

\section{Pierre Filiatrault, Marc Laplante et Sylvie Houle}

Volume 1, numéro 1, février 1982

L’hospitalité au Québec

URI : https://id.erudit.org/iderudit/1080861ar

DOI : https://doi.org/10.7202/1080861ar

Aller au sommaire du numéro

Éditeur(s)

Université du Québec à Montréal

ISSN

0712-8657 (imprimé)

1923-2705 (numérique)

Découvrir la revue

Citer cet article

Filiatrault, P., Laplante, M. \& Houle, S. (1982). L'attitude des Québécois envers les touristes : principaux résultats d'un sondage exploratoire. Téoros, 1(1),

6-12. https://doi.org/10.7202/1080861ar d'utilisation que vous pouvez consulter en ligne. 


\title{
L'attitude des Québécois envers les touristes
}

\author{
Principaux résultats d'un sondage exploratoire \\ par Pierre Filiatrault, Marc Laplante et Sylvie Houle
}

\begin{abstract}
Des interrogations nouvelles
En tourisme, comme en bien d'autres domaines, la société québécoise vient à peine de prendre en main son développement. En quelques années, elle a jeté les bases d'une politique sociale du tourisme, conçu des plans d'aménagement à long terme, rebåti son image aux yeux des visiteurs étrangers, etc. De tels changements devaient entraîner certaines remises en question d'idées reçues.
\end{abstract}

En mai 1980, une association, regroupant quelques centaines d'intervenants en tourisme, décidait de consacrer son congrès annuel au thème de l'hospitalité des Québécois. L'Association technique du tourisme invitait ses membres a s'interroger sur la tradition québécoise d'hospitalité. Dix ans plus tôt, quiconque aurait osé s'inquiéter de cette valeur sure du Québec n'aurait pas trouvé d'auditoire.

Le sondage, entrepris par le module de gestion et intervention touristiques de I'UOAM et résumé dans les pages qui suivent, est ambitieux malgré son caractère exploratoire. II vise prioritairement l'analyse des attitudes des Québécois envers les touristes. Qui parle d'attitudes réfère habituellement à une réalité complexe et difficilement observable. Une attitude, c'est une prédisposition à agir de telle ou telle façon, en quelque sorte une habitude de la pensée, une accoutumance a juger et à évaluer le réel. Face aux touristes, les Qué- becois sont-ils favorablement ou défavorablement disposés?

Favorablement, répondront spontanément la majorité des intervenants touristiques, en se référant à une tradition québécoise d'entregent, d'accueil et de visites parentales. Mais nos recherches historiques pour retrouver les bases de cette tradition furent peu fructueuses pour une bonne raison finalement car le tourisme au Québec est un phénomène contemporain... (à l'exception des Américains qui nous visient depuis si longtemps qu'ils ne sont presque plus de la "visite",..$)$.

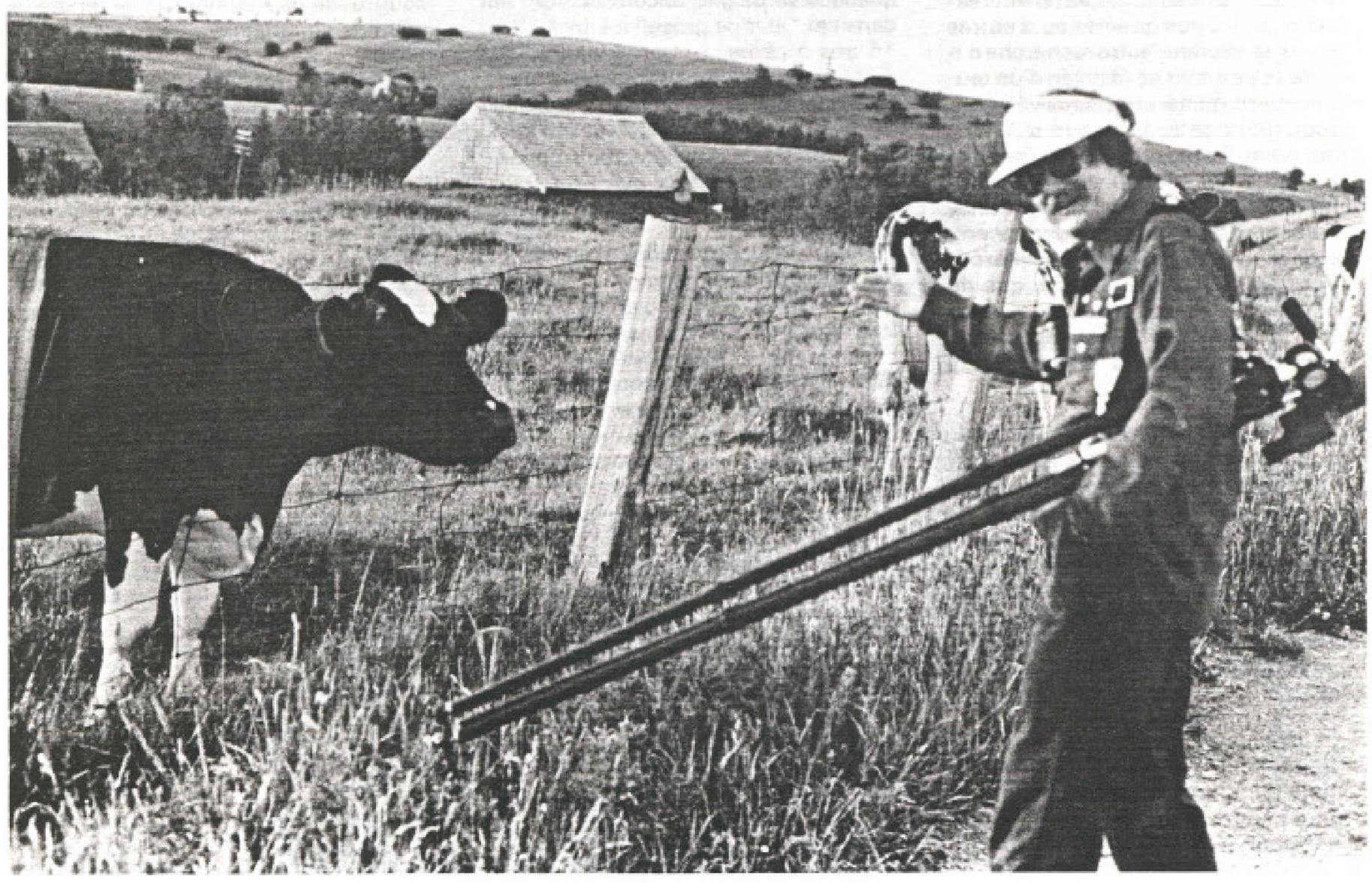


Ces considérations sur l'hospitalité et la tradition conduisent d'abord à constater la fragilité des affirmations, pourtant fréquentes, à propos de la prédisposition favorable des Québécois envers les touristes. Le sondage devait donc asseoir sur des bases plus solides nos intuitions et nos idées à ce sujet. II devait aussi nous faire avancer de quelques pas dans la compréhension de l'hospitalité touristique. Celle-ci est-elle pratiquée semblablement par tous, dans toutes les régions, à la ville comme à la campagne, dans les zones touristiques comme dans les coins plus isolés? Certes non. Alors, quelle est la situation de la pratique hospitalière au Québec en 1981 ?

Telles sont les questions qui ont décidé I'UQAM et I'A.T.T. à entreprendre une étude des attitudes des Québécois envers les touristes auprès d'un échantillon représentatif de la population.

\section{Qu'est-ce que I'hospitalité?}

Concept central pour le sondage, l'hospitalité est une notion difficile à saisir. Elle se manifeste extérieurement par certains gestes typiques comme tendre la main, dire bonjour, donner des informations, être poli et courtois, etc. II s'agit alors des gestes de civilité, de bon voisinage, ou d'amitié générale que quiconque peut manifester envers un autre, y compris envers un étranger, sans être foncièrement hospitalier. Cette hospitalité qu'encouragent plusieurs campagnes publicitaires actuelles constitue la base minimale: en deça, nous trouvons l'indifférence et bientồt le rejet, sinon l'agressivité.

L'hospitalité serait alors plus que la simple politesse coutumière entre gens civilisés. Nous avons fait I"hypothèse que I'hospitalité commence quand se manifeste un réel intérêt pour l'Autre en général. Appliquée au tourisme, cette conception signifie qu'un Québécois hospitalier serait un individu ouvert et curieux, reconnaissant dans le touriste une personne intéressante à connaître. En d'autres mots, si le touriste n'apparaît pas intéressant au point de départ, avant tout contact particulier et personnel, il sera difficile d'être hospitalier envers lui.

Cette acceptation de l'hospitalité nous a semblé conforme au sens littéral de ce mot et à son étymologie. Selon le Robert, l'usage actuel du mot hospitalité réfère à la "libéralité qu'on exerce en recevant quelqu'un sous son toit, en le logeant gratuitement". Et, par extension, le mot désigne "S/action de recevoir chez soi. d'accueillir avec bonne grăce $^{* \text {. ... }}$
Comment accueillir avec bonne grâce sans trouver un intérềt personnel dans cet accueil? Jadis, aux temps anciens, I'hospitalité était un devoir envers les pauvres, les indigents, les pellerins de passage, les quêteux, etc. Elle s'apparentait à la charité. Cette conception n'a plus beaucoup de sens aujourd'hui, surtout si on parle d'hospitalité envers les touristes qui n'ont rien des indigents.

En suivant cette piste de l'hospitalité concue comme une manifestation d'intérèt pour l'Autre ou pour le Visiteur. nous devons alors imaginer divers niveaux de cet interrèt. Recevoir un touriste sous son toit, partager sa nourriture ou l'héberger gratuitement sont des actes exigeants qu'on ne posera pas spontanément envers le premier visiteur rencontré dans la rue. Mais on peut prendre du temps pour jaser avec un touriste; on acceptera peut-être aussi de lui servir de guide durant quelques heures, etc.

En d'autres mots, les manifestations de l'hospitalité peuvent être très variées et se répartir entre deux pôles selon le degré d'implication du visité: d'un côté. les formes élémentaires de la politesse et de l'autre, l'intéressement fort qui consiste par exemple, à inviter à diner ou à loger chez soi un visiteur.

Pour les besoins du sondage, nous avons élaboré une échelle d'attitudes. c'est-â-dire, un outil susceptible de mesurer ces divers degrés d'intéressement et d'acceptation des Québécois envers les touristes. Les cinq propositions suivantes furent introduites dans le questionnaire et, pour chacune, nous demandions à l'interviewé s'il était parfaitement d'accord, d'accord, en désaccord ou tout à fait en désaccord avec elle.

A) Quand je vois un touriste qui cherche son chemin, je l'aide à se retrouver

B) Quand j'en ai l'occasion, j'aime prendre du temps pour jaser avec les touristes

C) Si jen avais I'occasion, j'irais avec un touriste visiter ma ville ou ma région

D) Parfois, on devrait inviter un touriste à diner pour lui faire connaître notre cuisine régionale

E) En certaines occasions, j'accepterais de loger un touriste gratuitement un soir chez moi.
Comme on le constate, ces propositions représentent des difficultés croissantes: il faut plus d'efforts, donc un plus grand intérêt, pour loger un touriste chez soi que pour lui aider à retrouver son chemin. Logiquement, donc, on devrait trouver des pourcentages d'accord plus élevés pour la proposition A) et de moinsen moins d'efforts ensuite pour les propositions B, C, D et E. Nous vérifierons cette hypothèse au paragraphe suivant. Précisons ici que ces propositions furent présentées de façon mélangée dans le questionnaire pour que le répondant ne perçoive pas la gradation. De plus, nous avons formulé également cinq propositions "négatives" envers les touristes pour susciter des réactions davantage émotives. Ces cinq propositions négatives s'échelonnent selon une gradation allant de l'indifférence ("Je préfére ne pas me mêler aux touristes") au rejet ("A mon avis. les touristes feraient mieux de rester chez eux ${ }^{\prime \prime}$ ). On comprendra que ces formules négatives visaient à permettre à certains répondants d'exprimer librement leur's malaises envers les touristes. En même temps, puisqu'elles étaient intercalées entre les propositions positives, elles empêchaient toute accoutumance chez l'interviewé.

Faut-il souligner ici, avant d'entreprendre la présentation des résultats, que cette échelle d'attitude n'est qu'un premier essai dans ce domaine. Elle n'a pas été testée avant le sondage qui n'est. lui-même, qu'un pré-test pour une éventuelle recherche systématique sur I'hospitalité touristique. 
Les Québécois sont favorablement disposés envers les touristes

Le tableau 1, ci-dessous, confirme d'abord que les Québécois, globalement considérés, se disent hospitaliers: $69.3 \%$ sont d'accord ou parfaitement d'accord avec la plus exigeante des propositions concernant l'attitude hospitalière: loger un touriste chez soi gratuitement en certaines circonstances.

Ce premier résultat tend à valider également l'échelle d'attitudes utilisée. Ceci est plus évident si on ne considère que la première colonne du tableau 1: les pourcentages de répondants parfaitement d'accord avec les propositions. Avec 4 possibilités de réponse seulement (deux pour accord et 2 pour désaccord) l"interviewé le moindrement favorable se dira d'accord avec une proposition. Cependant, s'il choisit la réponse: "parfaitement d'accord" , c'est pour manifester clairement son acceptation de l'idée proposée. Cette nuance importante s"exprime avec évidence par la proposition $E_{\text {: }} 60.2 \%$ sont d'accord pour loger gratuitement chez eux un touriste mais $9.1 \%$ seulement sont tout à fait d'accord avec cette idée. La réponse: "d"accord" apparait donc comme un accord de principe alors que la réponse "parfaitement d'accord" exprime davantage un consentement de fait. Á une exception près (la proposition B), I'accord de principe oscille entre $60 \%$ et $63.5 \%$ et c'est l'accord de fait qui différencie les répondants.

Le tableau 2 présente la distribution des réponses aux propositions négatives. Les résultats sont éloquents: les gens parfaitement en accord avec elles représentent au maximum, $1.6 \%$ de la population. La réponse "tout à fait en désaccord"' exprime mieux le carac= tère sympathique des Québécois.

\begin{tabular}{|c|c|c|c|c|}
\hline \multirow{3}{*}{ Propositions } & \multicolumn{3}{|c|}{$\begin{array}{l}\text { Tableau } 1 \\
\text { Pourcentage des répondants } \\
\text { ament d'accord et d'accord avec les propositions } \\
\text { ant des attitudes favorables envers les touristes. }\end{array}$} & \multirow[b]{2}{*}{$\begin{array}{c}\text { Nombre } \\
\text { de cas }\end{array}$} \\
\hline & $\begin{array}{l}\text { Parfaitement } \\
\text { d'accord }\end{array}$ & D'accord & $\begin{array}{l}\text { Total } \\
\text { Accord }\end{array}$ & \\
\hline & $\%$ & $\%$ & $\%$ & $(\mathbb{N})$ \\
\hline A: donner information & 37.5 & 61.1 & 98.6 & $(650)$ \\
\hline B: jaser & 20.6 & 73.8 & 94.4 & (649) \\
\hline C: servir de guide & 15.4 & 62.6 & 78.0 & (636) \\
\hline D: inviter à diner & 13.4 & 63.5 & 76.9 & (625) \\
\hline $\begin{array}{l}\text { E: loger chez soi } \\
\text { gratuitement }\end{array}$ & 9.1 & 60.2 & 69.3 & (613) \\
\hline
\end{tabular}

Tableau 2

Pourcentage des répondants en accord et en désaccord avec les propositions exprimant des attitudes négatives envers les touristes

\begin{tabular}{|c|c|c|c|c|c|c|}
\hline Propositions & $\begin{array}{l}\text { Parfaitement } \\
\text { d'accord }\end{array}$ & Accord & Désaccord & $\begin{array}{l}\text { Tout à fait } \\
\text { désaccord }\end{array}$ & Total & $\begin{array}{c}\text { Nombre } \\
\text { de cas }\end{array}$ \\
\hline & $\%$ & $\%$ & $\%$ & $\%$ & $\%$ & $(\mathbf{N})$ \\
\hline F: les endurer & 1.2 & 18.2 & 58.9 & 21.7 & 100 & $(642)$ \\
\hline G: Pas se mêler & 1.6 & 12.8 & 62.7 & 22.9 & 100 & $(625)$ \\
\hline H: Touristes ne respectent rien & 0.5 & 12.6 & 72.7 & 14.1 & 100 & $(587)$ \\
\hline I: Plus d'ennuis que d'avantages & 0.0 & 6.1 & 69.5 & 24.4 & 100 & (619) \\
\hline J: Rester chez eux & 1.1 & 2.3 & $\quad 47.4$ & 49.2 & 100 & (653) \\
\hline
\end{tabular}

F: "Les touristes sont rarement interessants mais il faut bien les endurer"

$\mathrm{G}$ : "Je preflere ne pas me méler aux touristes"

$\mathrm{H}$ : "Trop souvent, les touristes ne respectent rien"

l: "Les touristes m'apportent plus d'ennuis que d'avantages"

$\mathrm{J}$ : "A mon avis, les touristes feraient mieux de rester chez eux". 
Auparavant, une remarque d'ordre méthodologique s'impose. L"échantillon de 665 répondants nous autorise à faire des tableaux croisés avec deux variables (par exemple: I'attitude hospitalière selon les groupes d"âge) mais il est trop petit pour nous permettre de considérer a la fois plus que deux variables. Or, une telle procédure est indispensable pour éviter d'établir de fausses corrélations. Par exemple, nous avons constaté que les gens de 18 à 25 ans sont les plus favorables a la proposition E (loger quelqu'un chez soi): d'autre part, nous trouvons également que les gens du Coeur du Québec sont également les plus favorables à cette même proposition. Comment conclure? Est-ce l'âge qui influence la réponse favorable? Est-ce le fait d'habiter entre deux grands centres urbains? Si nous pouvions, en un seul tableau statistique, répartir les répondants a la fois selon leur âge et leur lieu de résidence, nous saurions quels rapports existent entre ces deux facteurs. L'échantillon est trop petit pour autoriser une analyse multivariée de ce genre. Nous ne retiendront donc que des tableaux à double entrée et nos commentaires seront prudents.

Pour cette me̊me raison, nous avons dû regrouper des catégories (d'âge, de scolarité, de revenus, etc.) qu'il eut été intéressant d'examiner en classes plus $\mathrm{fi}$ nes. Cette limite affecte surtout la distribution des réponses par région. Initialement, nous visions à respecter au moins le découpage du Québec en 10 régions administratives. (Idéalement, un sondage de ce genre devrait être valable au niveau de chacune des 18 régions touristiques du Québec). En fait, faute de temps et surtout de moyens financiers, certaines régions touristiques étaient sous-représentées dans l'échantillon. L'analyse statistique est invalide sans un nombre minimum de cas.
Le découpage du territoire finalement retenu est le suivant:

Nombre

de cas

Région est: Gaspésie, Bas

St-Laurent, Côte Nord, Sague-

nay, Lac St-Jean ................... . . . . 79

Region ouest: Outaouais, Nord-

Quest Québécois . ...................... 74

Région Montréal: Montréal, Rive

Nord, Rive Sud . . . . . . . . . . . . . . . . 237

Région Coeur: Mauricie, Can-

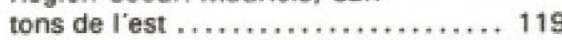

Région Québec: Québec, Char -

levoix, Portneuf, Beauce ............ 155

Nous avons regroupé, au tableau 3 , toutes les données nécessaires à l'analyse des relations entre les attitudes des Québécois envers les touristes et sept facteurs explicatifs susceptibles de faire apparaitre des variations de ces attitudes.

La proposition A (donner de l'information) est agréée, au total, par $37,4 \%$ de la population. Ce pourcentage varie de façon significative, ( 2 ) selon les régions, les habitudes de voyage, la langue et l'âge. Le pourcentage des gens "parfaitement d'accord" avec $A$ est nettement plus élevé dans la région Ouest ( $55.6 \%)$ et remarquablement plus bas dans la région de Québec. Les gens du Coeur du Québec sont également très en accord avec cette proposition. Les personnes qui ont fait plusieurs expériences de voyage (de 5 jours et plus hors de leur région) se disent nettement plus favorables que les autres à la proposition $\mathrm{A}$ : en multipliant les voyages on apprend peut-être à apprécier la valeur d'un bon informateur quand on cherche son chemin. Les gens qui, en plus du français. parlent couramment une autre langue se disent favorables à la proposition $\mathrm{A}$ plus que les unilingues français. Etre polyglotte prédispose peut-être au rôle d'informateur. Enfin, les personnes de 56 ans et plus semblent plus disponibles que les autres pour dépanner un touriste qui cherche son chemin. S'agitil de gens ayant plus de temps libre? Ou encore, ces personnes plus âgées sontelles particulièrement attentives à ce besoin d'être bien renseigné? Dans l'ensemble, la forme d'hospitalité la plus simple: aider un touriste à retrouver son chemin, ne semble pas propre à certains sous-groupes particuliers de la population québécoise. Les variations les plus grandes à ce propos s'observent entre les régions du Québec: dans deux territoires, fortement fréquentés par les touristes: Québec et l'est du Québec (qui comprend, dans notre cas, la Côte Nord et le Saguenay-Lac StJean en plus de la péninsule gaspésienne), la prédisposition de la population à aider le touriste égaré semble moins forte. $Y$-a-t-il corrélation entre cette exposition aux touristes et cette attitude? Pour répondre affirmativement sans hésitation, il faudra que nos résultats soient acceptables au niveau des régions plus particulières.

La proposition B (jaser avec des touristes) est très peu influencée par l'une ou l'autre des 7 variables explicatives, comme on peut le constater au tableau 3. seconde colonne, Dans I'ensemble. $20.7 \%$ de la population se dit parfaitement d'accord avec elle et ce pourcentage ne varie de façon significative qu'avec 2 facteurs: la langue parlée et le revenu. Une fois de plus, les unilingues français se disent moins favorables à B mais, pour jaser, il faut peut-être savoir s'exprimer en d'autres langues que la sienne. Pourquoi les gens qui disposent de revenus annuels au-dessus de $\$ 20,000$. sont-ils plus d'accord avec cette proposition? L'enquête nous a montré que les gens plus riches sont aussi plus instruits et ont plus voyagé. Est-ce que ces facteurs sont reliés à la proposition B? Si oui, c'est I'accumulation de ses facteurs (revenus, instruction, profession, expérience de voyage) qui favorise la réponse positive à la proposition $\mathrm{B}$.

Considérées globalement, les propositions $C$ (faire visiter sa ville ou sa région) et $\mathrm{D}$ (inviter à diner) sont à peu près également acceptées par les différents sous-groupes du Québec. Les variations observées entre les régions, entre les gens qui ont plus ou moins voyage. etc. ne sont pas significatives. La seule corrélation concerne les gens dont les revenus annuels dépassent $\$ 20,000$.: ils sont proportionnellement plus nom breux à accepter d'inviter un touriste à diner pour lui faire connaître la cuisine régionale. Dans ce cas-ci, la différence des revenus peut, à elle seule, influencer l'attitude. 
Comme on pouvait s'y attendre, c'est la proposition E (loger chez soi un soir gratuitement un touriste) qui entraine le plus grand nombre de variations selon les sous-groupes de la population. Elle est plus acceptée au Coeur du Québec et moins, dans la région Ouest. Les personnes n'ayant jamais voyagé ( 5 jours et plus) hors de leur région l'acceptent moins facilement. Les gens plus instruits (16 ans et plus d'études) sont davantage favorables a cette forme exigeante d'hospitalité. Les femmes l'agréent plus que les hommes et les jeunes de 18 à 25 ans, plus que les gens des autres groupes d'âge.
Seule une analyse multivariée, permettant de considérer à la fois l'influence respective de deux ou plusieurs facteurs explicatifs, mettra en lumière les rapports complexes qui apparaissent actuellement entre ces diverses variables.

Nous pouvons toutefois procéder à une lecture différente des données du tableau 3. Considérons l'ensemble des 5 propositions ( $A$ a E) et examinons les grandes différences selon chacune des variables. Ainsi, I'influence de la région semble réelle. La région de Québec obtient des pourcentages inférieurs à la moyenne québécoise pour les 5 propositions. Par opposition, la région du Coeur du Québec montre des pourcentages supérieurs à cette même moyenne. Pour mieux saisir ces différences globales, nous pouvons les exprimer à I'aide d'une mesure (3) que nous avons élaborée à partir des données du tableau 3 .

Tableau 3

Pourcentage des répondants "parfaitement d'accord" avec chacune des propositions favorables aux touristes, selon diverses variables. (1)

Propositions

\begin{tabular}{|c|c|c|c|c|c|}
\hline & & & & & \\
\hline & A & B & C & D & $\mathbf{E}$ \\
\hline Population totale & 37.4 & 20.7 & 15.4 & 13.5 & 9.2 \\
\hline Régions: Est & 24.4 & 19.2 & 13.9 & 11.8 & 9.6 \\
\hline Ouest & 55.6 & 19.2 & 20.0 & 11.3 & 5.6 \\
\hline Montréal & 42.6 & 24.3 & 15.1 & 17.5 & 9.6 \\
\hline Coeur & 46.6 & 26.7 & 21.9 & 14.5 & 11.9 \\
\hline Quếbec & $\begin{array}{c}20.5 \\
\text { (S) }\end{array}$ & 11.6 & 9.5 & 7.9 & $\begin{array}{l}7.9 \\
\text { (S) }\end{array}$ \\
\hline
\end{tabular}

Voyages de 5 jours et plus hors région:

$\begin{array}{ll}\text { Non } & 31 \\ 1 \text { fois } & 29 \\ 2 \text { fois } & 25 \\ 3 \text { fois et plus } & 45\end{array}$

$\begin{array}{rrrrr}31.7 & 15.2 & 11.4 & 9.6 & 3.9 \\ 29.2 & 21.6 & 15.2 & 12.9 & 9.1 \\ 25.0 & 15.4 & 10.4 & 9.1 & 13.8 \\ 45.9 & 24.3 & 18.6 & 16.6 & 10.9\end{array}$

(s)

Nombre d'années d'études:

0.9 ans

10-12 ans

13-15 ans

16 ans et plus
37.9

36.8

35.7

41.7
20.3

18.6

25.4

20.3
18.5

13.0

15.2

16.0
13.8

12.3

14.8

14.5
9.8

6.2

10.5

14.1

(S)

$$
\begin{aligned}
& \text { Langue parlée couramment: } \\
& \text { Français } \\
& \text { Français et autres }
\end{aligned}
$$

$34.1 \quad 17.2$

17.2

15.3

11.5

9.0

43.8

(S)

15.7

17.1

Revenus annuels

$\$ 20,000$ moins

$\$ 20,000$ plus
39.4

34.0
19.0

22.6

(S)

37.8

37.8

36.2

29.6

41.7

38.4

$36-45$ ans

46.55 ans

56 ans, plus

23.2

18.8
45.7

(s)
15.1

15.8

8

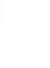

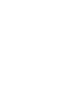

10

15.1

(S)

9.9

7.8

$\begin{array}{rrr}12.3 & 11.8 & 6.7 \\ 17.2 & 14.4 & 10.6\end{array}$

(S)

$\begin{array}{ll}\text { Age: } & 18-25 \text { ans } \\ & 26-35 \\ & 36-45 \text { ans } \\ & 46-55 \text { ans } \\ & 56 \text { ans, plus }\end{array}$

21.5
19.3
15.9
26.4
22.8

22.8

2.8

25.2

(s)

$\begin{array}{rrr}15.8 & 12.6 & 12.2 \\ 9.8 & 13.3 & 7.5 \\ 14.7 & 11.0 & 7.3 \\ 15.1 & 18.1 & 9.5 \\ 25.2 & 15.6 & 10.2 \\ \text { (s) } & & \end{array}$

(S) 
Cette mesure qui s'apparenterait à un indice d'hospitalité, peut prendre théoriquement toutes les valeurs comprises entre 1 et 5. Pour I'ensemble des Québécois, cette valeur-indice est de 2.31 . Le graphique I ci-dessous fait ressortir la valeur de cet indice pour les régions et les divers sous-groupes de la population.

Par rapport à la valeur d'indice 2.31 pour I'ensemble du Québec, qui nous sert de point de référence, la région de Québec apparait clairement moins hos- pitalière, suivie de la région Est. Ces deux territoires sont les plus visités par les touristes. En comparaison, les gens du Coeur du Québec semblent très hospitaliers.

L'habitude des voyages influence aussi les attitudes: ceux qui ont beaucoup voyagé ont une valeur-indice de 2.74 contre 1.55 pour ceux qui n'ont pas voyagé hors de leur région.

Les différences de scolarité, de langue. de revenus et de sexe n'introduisent pas des variations importantes de l'indice d'hospitalité.

L'âge, enfin, semble influencer les attitudes. Les jeunes de 18 à 25 ans ont un indice d'hospitalité voisin de la valeur moyenne pour tous les Québécois; de 26 ans à 56 ans et plus, I'indice d'hospitalité s'accroît avec l'âge; avec une valeur-indice de 2.91 , les personnes de 56 ans et plus forment le groupe social le plus hospitalier au Québec.

\section{Graphique I}

Variations de l'indice d'hospitalité selon les régions, les habitudes de voyage et les sous-groupes de la population du Québec. Valeur minimale: 1 (peu hospitalier)

Valeur maximale: 5 (très hospitalier)

$\begin{array}{llllllllllllllllllllll}1 & 1.1 & 1.2 & 1.3 & 1.4 & 1.5 & 1.6 & 1.7 & 1.8 & 1.9 & 2.0 & 2.1 & 2.2 & 2.3 & 2.4 & 2.5 & 2.6 & 2.7 & 2.8 & 2.9 & 3.0 & 3.1\end{array}$

Population totale:

Régions:

Est

Ouest

Montréal

Coeur

Québec

Voyage de 5 jours et plus:

Non

1 fois

2 fois

3 fois et plus

Années d'études:

0 - 9 ans

10-12 ans

13-15 ans

16 ans et plus

Langue:

Français

Français et autres

Revenus:

$\$ 20,000$. ou moins

$\$ 20,000$. ou plus

Sexe:

Homme

Femme

Åge:

18-25 ans

26-35 ans

36-45 ans

46-55 ans

56 ans et plus

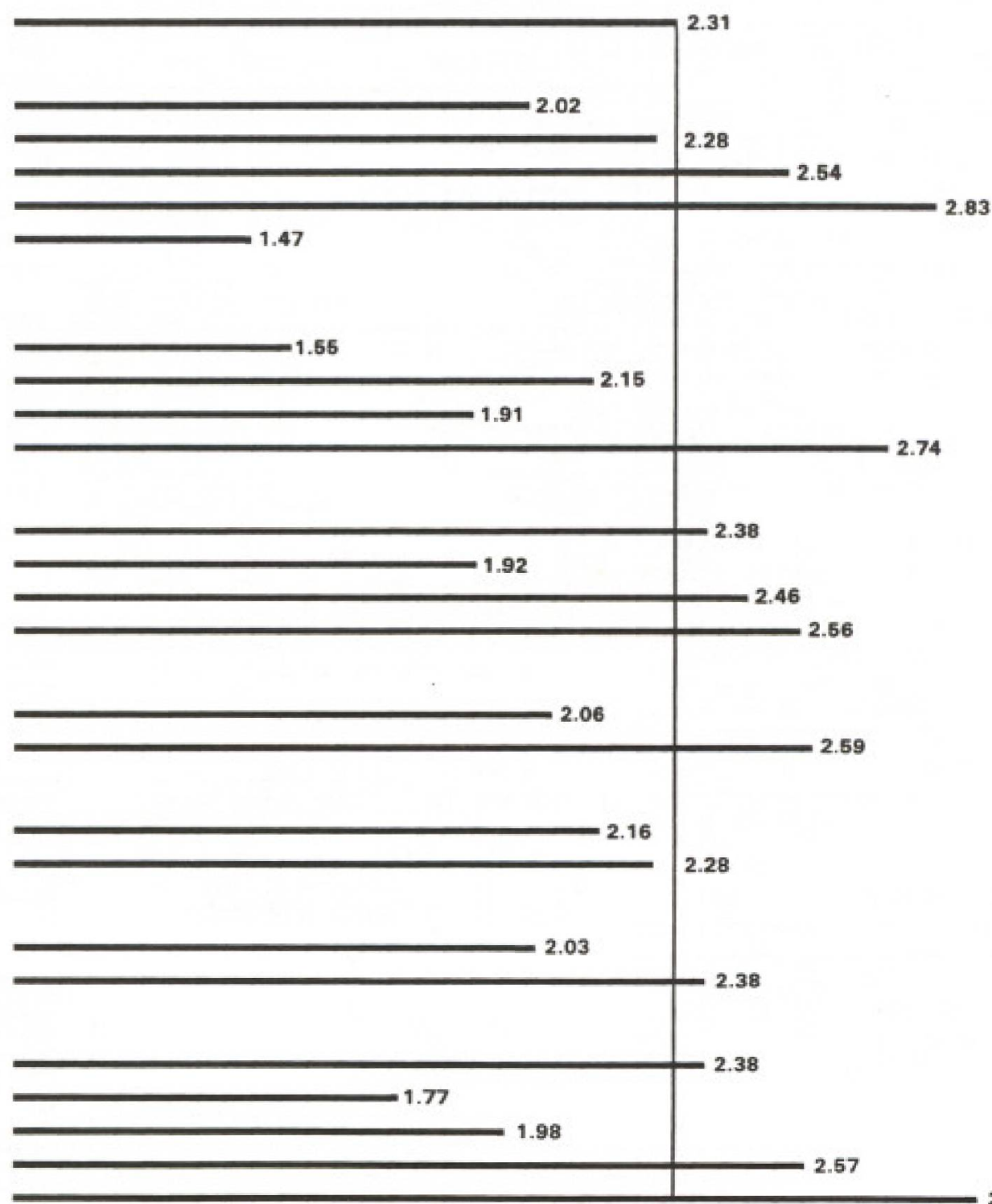

2.91 


\section{Conclusion}

En terminant cette analyse, on ne peut que souhaiter ardemment reprendre ce sondage avec un échantillon plus grand et plus solide au niveau des régions du Québec. De nombreuses questions se posèrent à nous à mesure que nous obtenions ces premiers résultats. Par exemple, si l'hospitalité s'accroît en fonction de l'âge, est-ce parce que les personnes plus âgées, libérées du rythme épuisant de la vie familiale, moins accaparées par le travail, sont plus disponibles et mieux organisées pour faire visiter, recevoir, prendre le temps de jaser, etc?

Ou bien, ces personnes appartiennentelles à une génération qui n"a pas connu, jeune, le tourisme de masse et qui a conservé pour les visiteurs et les étrangers une politesse, une courtoisie et mème une attitude traditionnelle d'hospitalité qui se pratiquait quand les touristes étaient de la "visite rare"? Si cette dernière hypothèse se vérifiait, il faudrait s'attendre à moins d'hospitalité dans l'avenir, quand seront disparus ceux et celles qui, aujourd'hui, la pratiquent encore intensivement. Par contre, si le sens de I'hospitalité se développe avec l'âge, comme la population du Québec vieillit, on trouvera, dans l'avenir, une proportion plus grande de gens très hospitaliers.

Les différences observées au niveau des régions ne peuvent être considérées sans réserve actuellement. Nous savons que, d'un territoire à l'autre, la population varie selon l'âge, le niveau d'études, le revenu, etc. Une analyse multivariée nous permettrait de tirer au clair ces questions. Pour les intervenants, nous devrons aussi introduire de nouvelles variables explicatives, plus spécifiques, pour tenter d'expliquer mieux les différences d'attitudes envers les touristes. Par exemple, nous avons constaté qu'à force de voyager, les individus acquièrent des expériences qui influencent leur indice d'hospitalité. Est-ce que les gens très "sociables", qui aiment recevoir du monde chez eux. sont plus favorablement disposés envers les touristes? Est-ce que la manière dont on utilise ses temps libres est en relation avec l'intérêt pour les touristes?

Si des questions comme celles-ci demeurent présentement sans réponses. après ce premier sondage, nous savons au moins que l'hospitalité envers les touristes est plus que cette pratique. si simple en apparence, qui consiste à sourire et à tendre la main de l'amitié.
ANNEXE 1

\section{La méthodologie du sondage}

\section{Le questionnaire}

Le questionnaire a été élaboré par des professeurs et des étudiants du module de gestion et intervention touristiques de I'UQAM composant l'équipe de recherche. Un pré-test a été réalisé et les corrections nécessaires ont été apportées au questionnaire original.

\section{L'échantillon}

L'échantillon est composé de numéros de téléphone tirés au hasard de façon systématique dans des bottins téléphoniques des dix régions administratives du Québec. L'échantillon a été tiré par le Centre de sondage de l'Université de Montréal. L'échantillon de départ était de 2706 numéros de téléphone. Le nombre des répondants est de 665 .

\section{La collecte des données}

Les entrevues téléphoniques ont été effectuées entre le $1 \mathrm{er}$ et le $10 \mathrm{mai}$ 1981 par les étudiants du module de gestion et intervention touristiques de I'UQAM à partir des dix régions administratives du Québec avec le support de plusieurs associations touristiques régionales.

\section{Le taux de réponse}

Le taux de réponse aux entrevues fut le suivant:

$\begin{array}{lrr} & \text { Nombre } & \% \\ \text { Entrevues complétées } & 665 & 24.6 \% \\ \text { Refus dé répondre } & 335 & 12.3 \% \\ \text { Service interrompu } & 668 & 24.6 \% \\ \text { Unités inéligibles } & 255 & 9.4 \% \\ \text { Langue étrangère } & 102 & 3.8 \% \\ \text { Pas de réponse } & 513 & 18.9 \% \\ \text { Incomplet } & 172 & 6.4 \% \\ \text { Total: } & 2,706 & 100.0 \%\end{array}$

\section{La marge d'erreur statistique}

La marge d'erreur statistique sur les réponses du questionnaire est de $4.1 \%$ lorsqu'une proportion est de $50 \%$ au niveau de confiance de $95 \%$.

\section{Le traitement des données}

Après avoir été codifiées par l'équipe de recherche et transcrites sur cartes perforées, les données furent analysées à l'aide du programme SPSS (Statistical Package for the Social Sciences) sur l'ordinateur CYBER de I'Université du Québéc à Montréal.

\section{ANNEXE 2}

Autres résultats significatifs du sondage

a) $25.6 \%$ des Québécois n'ont jamais fait un voyage de vacances de 5 jours et plus en dehors de leur région.

b) $10 \%$ des répondants ont voyagé seuls lors de leur plus récent voyage et $14.3 \%$ ont fait un voyage organisé.

c) $42.5 \%$ des gens affirment avoir fait plusieurs rencontres avec des gens des pays ou de la région visités lors de leur dernier voyage.

d) $51.4 \%$ disent n'avoir jamais eu de contacts avec des touristes de passage dans leur région et $14.9 \%$ affirment en avoir eu souvent. $44.4 \%$ de ces contacts furent jugés superficiels, $29.7 \%$, occasionnels et $25.9 \%$, soutenus, personnels et durables.

e) $62.3 \%$ des répondants ont déjà fait une visite touristique de leur propre région. Plus les gens ont fait des expériences de voyage, hors de leur région, plus aussi ils ont visité leur région.

f) Plus une personne est scolarisée, plus elle dit avoir eu de nombreux contacts avec les gens durant ses voyages hors région et avec des touristes de passage dans sa propre région.

g) 3 Québécois sur 4 environ, estiment que I'hospitalité pratiquée dans les hôtels, restaurants, stations de service, kiosques d'information et services municipaux est bonne. $64.1 \%$ jugent bonne l"hospitalité dans les motels.

Notes

(1) Compte-tenu des remarques faites précédemment, les analvses statistiques né porteront que sur les répon dants qui se sont dits "parfaitement d'accord" avec chacune des propositions. On se souviendra toutefois que de forts pourcentages de gens se sont dits "d'accord" avec ces mémes proposition.

(2) Les relations statistiquement significatives, dans le cadre de ce sondage, sont celles dont les variations ine son pas dues au hasard. Nous avons accepte deux limites de confiance qui sont marquees, dans le tableau 3, par "S (P egale ou plus petit que .01) et " $s$ " (P compris entre, 0 . et .011; une relation marquede $\mathbf{S}$ esa plus forte qu'une à tre, marquée $s$

(3) Arbitrairement, nous avons attribue un poids de 1 a la proposition A. un poids de 2 a 8 . de 3 a C. de 4 a Det de 5 a E. Ceci exprime la difficulte croisssante des propositions.

Nous awons ens.uite multiplie le nombre des personnes ayant répondu "partaitement daccord" A chacune des propositions par ces valours arbitraires. Finalement. nous awons laif ks somme des nombres ainsi obtenus of nous l'awons diviste par le total des rependants. Par exemple, pour l'ensemble du Qubbec. nous avons: A: $243 \times 1+$ B: $134 \times 2$, C: $98 \times 3+$ D: $84 \times 4+$ E: $56 \times 5$ $1421=615=2.31$. Pour l'ansemble des Quebtcois ce nombre represente ce qu'on peut appeler: l'indice d'thomitalith. En calculant de mome leconcet info pher pour chacun des sous groupes do la population chacune des régions, nous pouvons faire des compa raisons. 